Chromium-compensated GaAs detector material and sensors

This content has been downloaded from IOPscience. Please scroll down to see the full text. 2014 JINST 9 C07011

(http://iopscience.iop.org/1748-0221/9/07/C07011)

View the table of contents for this issue, or go to the journal homepage for more

Download details:

IP Address: 131.169.233.159

This content was downloaded on 03/02/2015 at 12:26

Please note that terms and conditions apply. 


\section{Chromium-compensated GaAs detector material and sensors}

\section{Budnitsky, ${ }^{a}$ A. Tyazhev, ${ }^{a, 1}$ V. Novikov, ${ }^{a}$ A. Zarubin, ${ }^{a}$ O. Tolbanov, ${ }^{a}$ M. Skakunov, ${ }^{a}$ E. Hamann, ${ }^{b, c}$ A. Fauler, ${ }^{b}$ M. Fiederle, ${ }^{b, c}$ S. Procz, ${ }^{b}$ H. Graafsma ${ }^{d}$ and S. Ryabkov ${ }^{e}$}

${ }^{a}$ Functional Electronics Laboratory, Tomsk State University, Lenin 36, Tomsk 634050, Russia

${ }^{b}$ FMF, University of Freiburg,

Friedrichstrasse 39, Freiburg 79098, Germany

${ }^{c}$ IPS, Karlsruhe Institute of Technology,

Hermann-von-Helmholtz-Platz 1, Eggenstein-Leopoldshafen 76344, Germany

${ }^{d}$ Deutsches Elektronen-Synchrotron (DESY),

Notkestrasse 85, Hamburg 22607, Germany

${ }^{e} X D i C o n$ LLC,

Uchebnaya street 8, office 221, Tomsk 634034, Russia

E-mail: antontyazhev@land.ru

ABSTRACT: Results obtained from numerical calculations of and experimental studies on the pulse height distribution inherent in ionizing radiation gallium arsenide sensors as a function of the design features of the devices and electrophysical characteristics of the detector material are presented. It is shown that the pulse height distribution is defined by the distribution pattern of the nonequilibrium charge carrier lifetime and by the electric field profile in the bulk of the sensor. Investigations on the detector sensitivity to X-ray energies in the range between 40 and $150 \mathrm{keV}$ were performed. The sensor polarization was found to produce only a marginal effect compensated by an increase in the bias voltage. Prototype pixel sensors measuring $256 \times 256$ and $512 \times 768$ pixels with a $55 \mu \mathrm{m}$ pitch and a $500 \mu \mathrm{m}$ thick sensitive layer were produced. The dependence of the photocurrent and count rate on the X-ray radiation intensity and bias voltage applied to the sensor was examined. In the $40-80 \mathrm{keV}$ energy range, the maximum count rate amounted to $800 \mathrm{kHz} /$ pixel for a negative sensor bias voltage of $800 \mathrm{~V}$. The sensors are demonstrated to provide spatial resolution varying with the pixel pitch and to enable high-quality X-ray images to be obtained.

KEYWORDS: X-ray detectors; Gamma detectors (scintillators, CZT, HPG, HgI etc)

\footnotetext{
${ }^{1}$ Corresponding author.
} 


\section{Contents}

1 Introduction 1

2 Experiment 1

2.1 Pulse height distribution 1

2.2 Count rate and X-ray sensitivity of GaAs:Cr sensors 3

2.2.1 Pad GaAs:Cr sensors 3

2.2.2 GaAs:Cr pixel sensor bonded to Timepix ASIC 4

2.2.3 Prototype GaAs:Cr imaging detectors 5

3 Conclusions $\quad 6$

\section{Introduction}

As discussed earlier [1-4], gallium arsenide compensated with chromium is a promising material for semiconductor detectors with X-ray photon energies ranging from 15 to $60 \mathrm{keV}$. The results of investigations on the variations in the pulse height distribution with the electrophysical characteristics of the detector material and in the output signal (photocurrent and count rate) with the X-ray intensity and bias voltage are presented.

\section{Experiment}

\subsection{Pulse height distribution}

One of the factors influencing the pulse height distribution, charge collection efficiency (CCE) and detector sensitivity is the nonequilibrium charge carrier (NCC) lifetime and its distribution through the thickness of the detector structure. Figure 1 shows the experimental dependence of the resistivity and photocurrent distributions through the detector structure and of the NCC lifetime on the coordinate position used in the spectrum calculations. Experimental samples of Type 1 and Type 2 were prepared from different parts of the bulk of a GaAs:Cr wafer. The NCC lifetime was determined experimentally from the CCE dependence on the bias voltage for the detector irradiation with $1 \mathrm{MeV}$ energy beta-particles. The NCC lifetime was estimated by means of experimental samples of Type 2 (figure 1) with a minimum gradient of the NCC lifetime. A detailed procedure employed to determine the mobility-lifetime products for electrons and holes is described elsewhere [5]. Low photocurrents in the near-surface layers of the detector material (figure 1) are due to short NCC lifetimes. This is caused by the nonstoichiometry of GaAs in the surface detector layers during high-temperature annealing. The defect layer thickness on each side of the wafer may be as large as $150 \mu \mathrm{m}$. Figure 1(a) suggests that in the case of fairly uniform resistivity distribution and hence of uniform electric field distribution, an increase in the photocurrent is due to an 


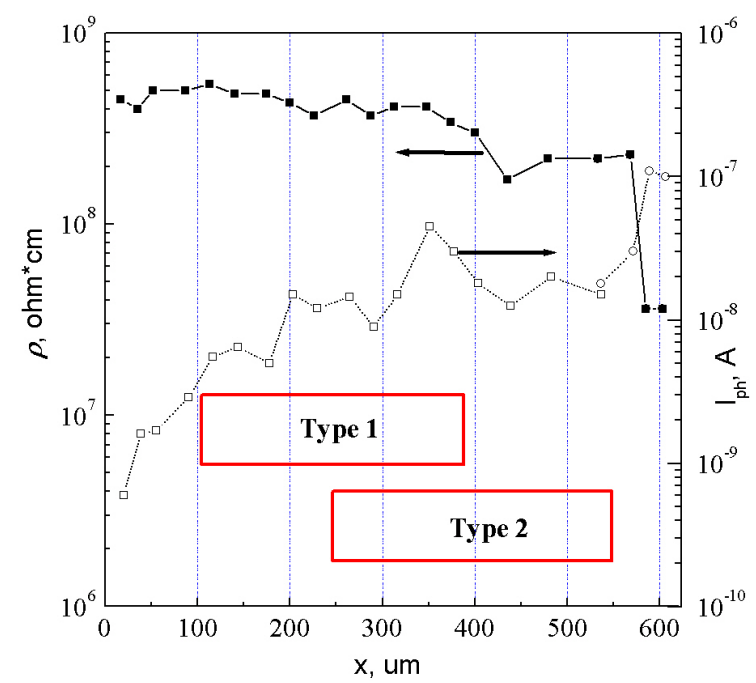

(a)

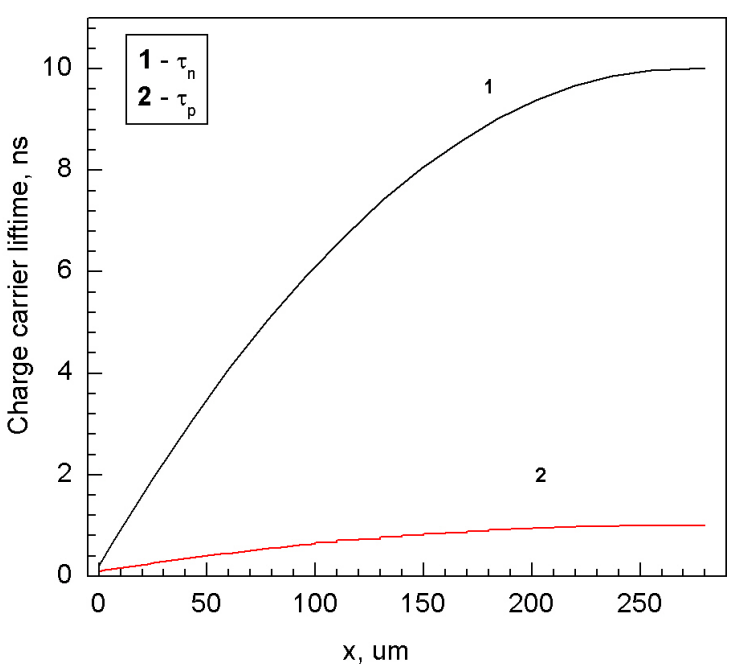

(b)

Figure 1. Resistivity $\rho$ and photocurrent $I_{\mathrm{ph}}$ distributions through the thickness of the high-resistivity GaAs:Cr wafer (a) and nonequilibrium charge carrier lifetime distribution (electrons $\tau_{n}$ and holes $\tau_{p}$ ) through the detector structure thickness $x$ (b).

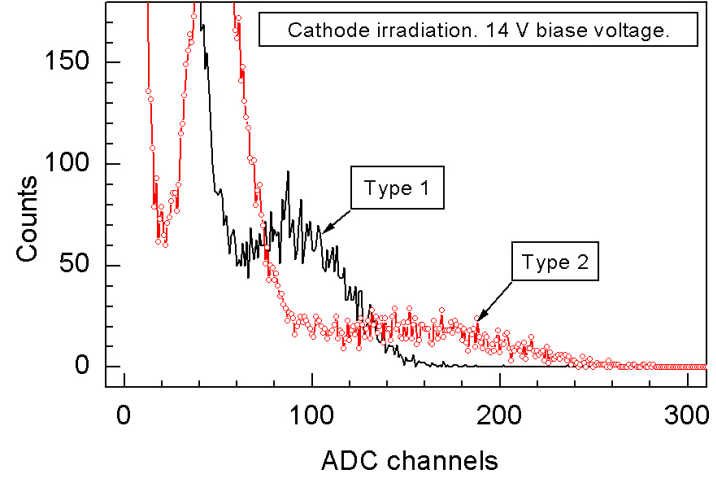

(a)

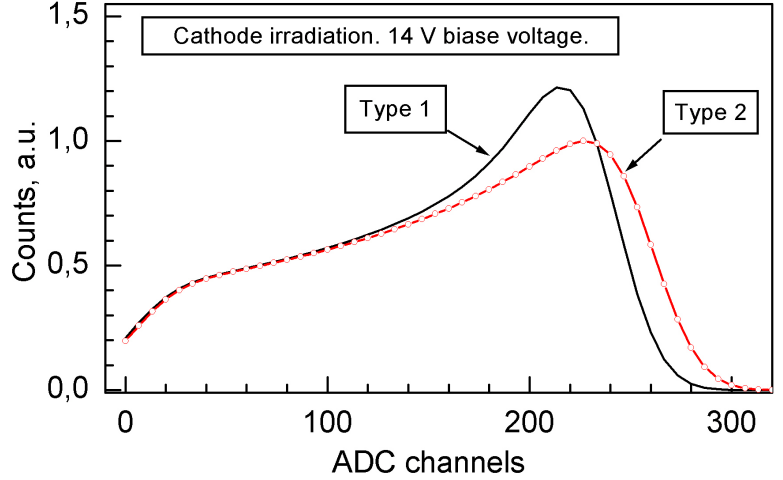

(b)

Figure 2. Experimental (a) and calculated spectra (b) of the detectors with uniform (Type 2) and nonuniform (Type 1) NCC lifetime distributions through the wafer thickness. The bias voltage at the irradiated contact is $14 \mathrm{~V}$. It should be noted that the dependence of the NCC lifetime on the coordinate position leads to a more distinct $60 \mathrm{keV}$ peak in the pulse height distribution and to a lower CCE for the same bias voltage. The difference in the pulse height distributions between the Type 1 and Type 2 structures decreases with increase in the bias voltage due to the linearization of the CCE dependence on the coordinate position.

increase in the NCC lifetime as the carriers move from the wafer surface $(x=0)$ to its depth. The NCC lifetime profile used in the spectrum calculations is illustrated in figure 1(b). Experimental (a) and calculated spectra (b) of the sensors (Types 1 and 2) irradiated with gamma photons with an energy of $60 \mathrm{keV}\left({ }^{241} \mathrm{Am}\right)$ are presented in figures $2-4$ for varying bias voltage. The computational procedure used for the pulse height distribution is outlined in [6]. 


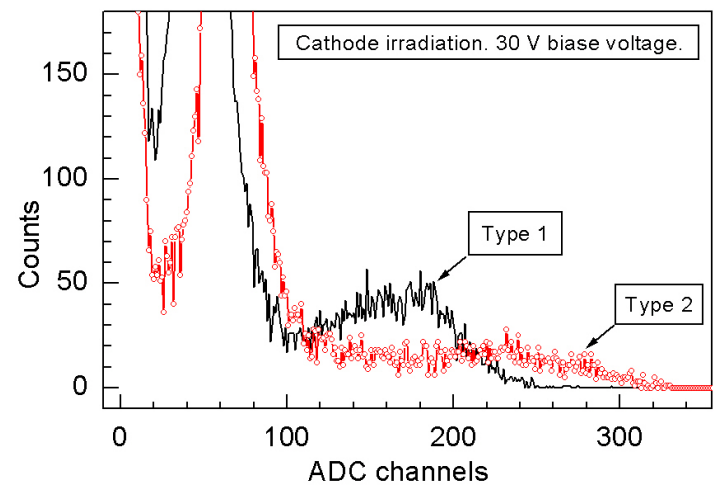

(a)

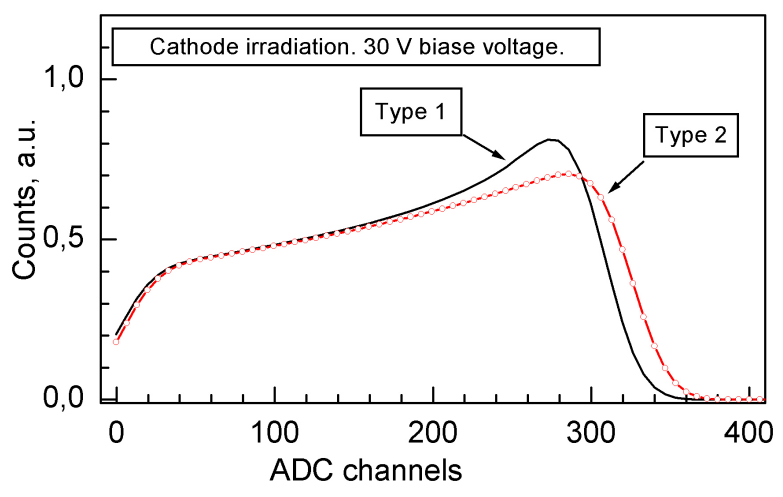

(b)

Figure 3. Experimental (a) and calculated spectra (b) of the detectors with uniform (Type 2) and nonuniform (Type 1) NCC lifetime distributions through the wafer thickness. The bias voltage at the irradiated contact is $30 \mathrm{~V}$.

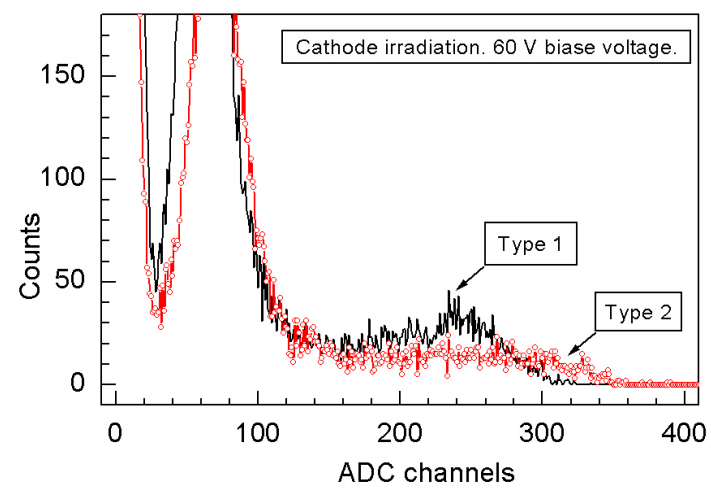

(a)

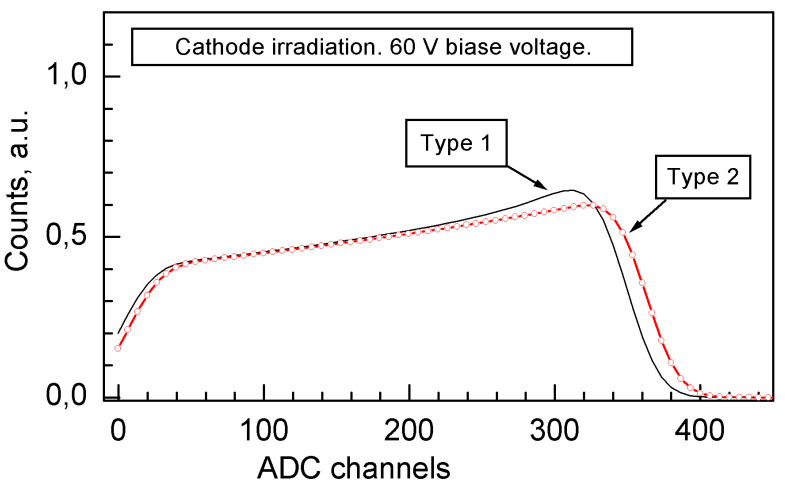

(b)

Figure 4. Experimental (a) and calculated spectra (b) of the detectors with uniform (Type 2) and nonuniform (Type 1) NCC lifetime distributions through the wafer thickness. The bias voltage at the irradiated contact is $60 \mathrm{~V}$.

Analysis of the data demonstrated in figures 2-4 leads us to suggest that the experimental and calculated spectra both with uniform and nonuniform NCC lifetime distributions over the sensitive layer thickness are in qualitative agreement.

\subsection{Count rate and X-ray sensitivity of GaAs: Cr sensors}

\subsubsection{Pad GaAs:Cr sensors}

A tungsten anode X-ray tube with an inherent filtration equal to that of a $4 \mathrm{~mm}$ thick Al filter served as an X-ray source. A $500 \mu \mathrm{m}$ thick pad sensor was placed in a metal housing with a window closed by a metallized mylar film. The resistance $R(110 \mathrm{k} \Omega)$ was connected in series with the detector. The voltage drop was measured by an oscilloscope. The experimental dependence of the photocurrent $\left(I_{\mathrm{ph}}\right)$ on the exposure rate $(\mathrm{ER})$ is presented in figure 5. Analysis of the results obtained shows that the detector sensitivity is higher for cathode irradiation (figure 5(b)) than in the case of anode irradiation (figure 5(a)), with other parameters being equal. The $I_{\mathrm{ph}}$ saturation 


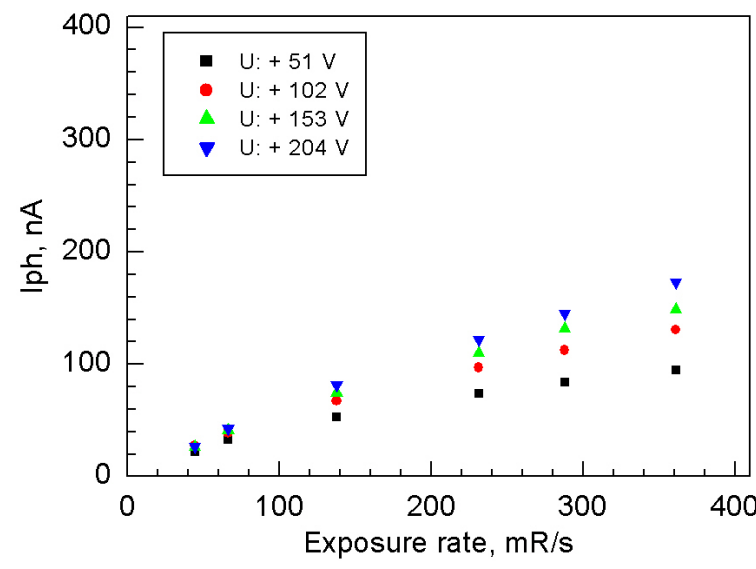

(a)

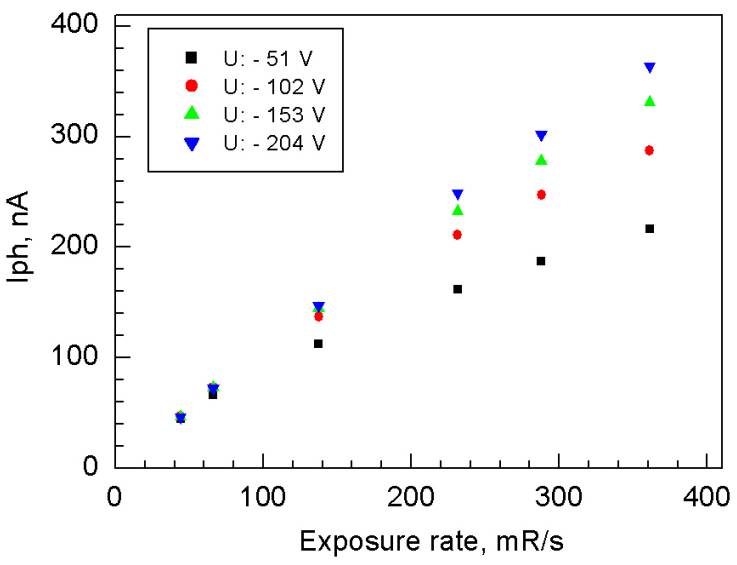

(b)

Figure 5. Dependence of the photocurrent $I_{\mathrm{ph}}$ on the bias voltage: anode (a) and cathode irradiation (b), an anode X-ray tube with $U=80 \mathrm{kV}$ and a GaAs:Cr sensor measuring $0.3 \times 0.3 \mathrm{~cm}^{2}$ with a sensitive layer thickness of $510 \mu \mathrm{m}$.

with increase in the DR is likely to be due to the sensor polarization. This is likely to be associated with uncompensated charge in the bulk of the sensor. As a consequence, the effective electric field strength in the sensitive region is decreased, with a corresponding reduction in the CCE. Notably, where the electric field strength reaches $4 \mathrm{kV} / \mathrm{cm}$, we get a linear dependence of $I_{\mathrm{ph}}$ on the DR for an intensity ranging from 0 to $400 \mathrm{mR} / \mathrm{s}$ and a tube voltage of $80 \mathrm{kV}$.

\subsubsection{GaAs: Cr pixel sensor bonded to Timepix ASIC}

Figure 6 demonstrates the test results obtained from prototype detectors based on a GaAs:Cr pixel sensor $(256 \times 256$ pixels, $55 \mu \mathrm{m}$ pitch) bonded to a Timepix chip. A tungsten anode X-ray tube served for an X-ray source, as in the case of pad sensors. It is evident from the figure that for a bias voltage of $-50 \mathrm{~V}$ and an DR of more than $140 \mathrm{mR} / \mathrm{s}$, the count rate decreases (figure 6(a)). This effect can be compensated by increasing the bias voltage up to $-200 \mathrm{~V}$. The results presented in figure 6 are in agreement with those shown in figure 5. It is pertinent to note that the operating bias voltage of a $500 \mu \mathrm{m}$ thick GaAs:Cr sensor is $-200 \mathrm{~V}$ (figure 7(a)). In this case, the count rate is no less than $100 \mathrm{kHz} /$ pixel.

Results of investigations on the maximum count rate for the GaAs: $\mathrm{Cr}+\mathrm{TPX}$ detector are given in figure 7(b). Analysis shows that for an X-ray flux intensity exceeding $3 \times 10^{6}$ photons/pixel $\times \mathrm{s}$, the increase in the maximum count rate is saturated. A conceivable reason for this effect is a limited Timepix chip speed of response $(0.5-1 \mathrm{MHz} / \mathrm{pixel})$ [7]. Nevertheless, in the energy range lying between 40 and $-80 \mathrm{KeV}$, the count rate of the GaAs:Cr Timepix detector amounts to $800 \mathrm{kHz} /$ pixel for a sensor bias voltage of $-800 \mathrm{~V}$. The most plausible reason for the disagreement between the experimental and the calculated maximum count rates (figure 7(b)) is the error in the estimation of the effective absorption coefficient of multispectral X-ray radiation. 


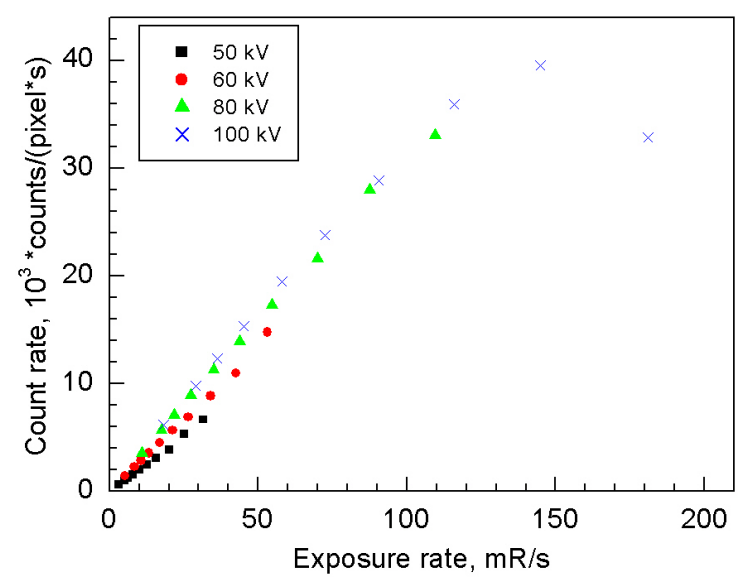

(a)

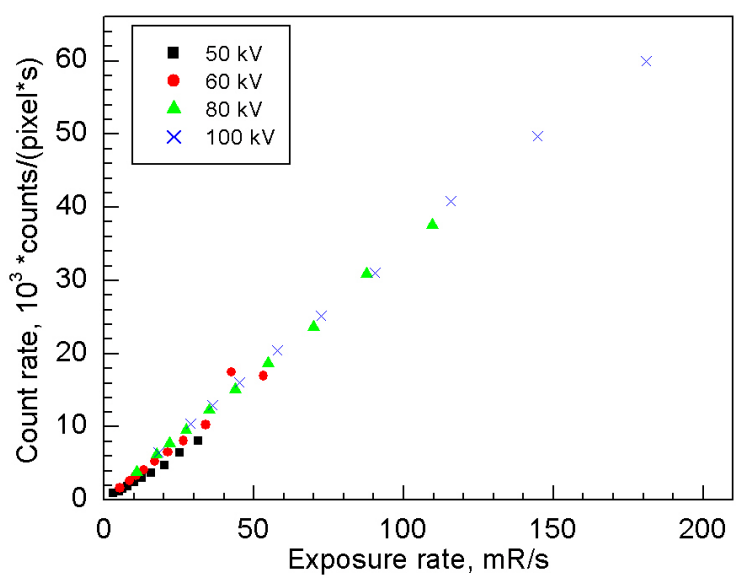

(b)

Figure 6. Dependence of the count rate on the exposure rate; cathode irradiation: -50 (a) and $-200 \mathrm{~V}$; a GaAs:Cr pad sensor measuring $0.3 \times 0.3 \mathrm{~cm}^{2}(\mathrm{~b})$ and thickness of $510 \mu \mathrm{m}$.

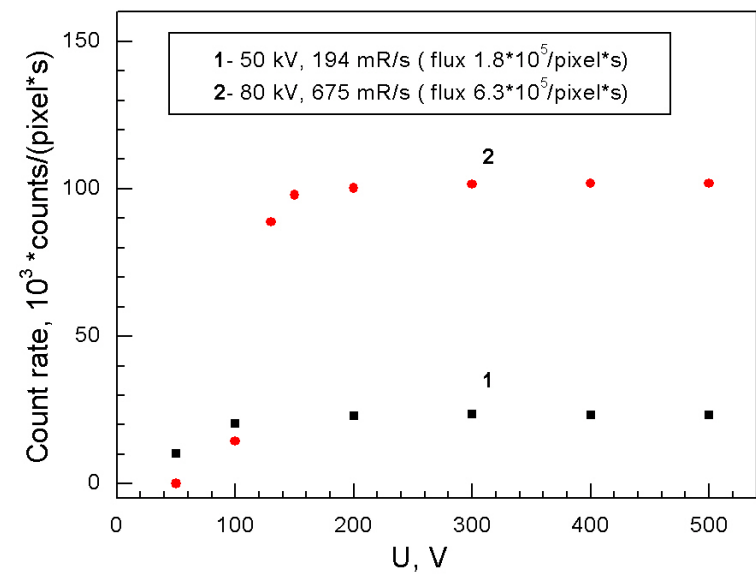

(a)

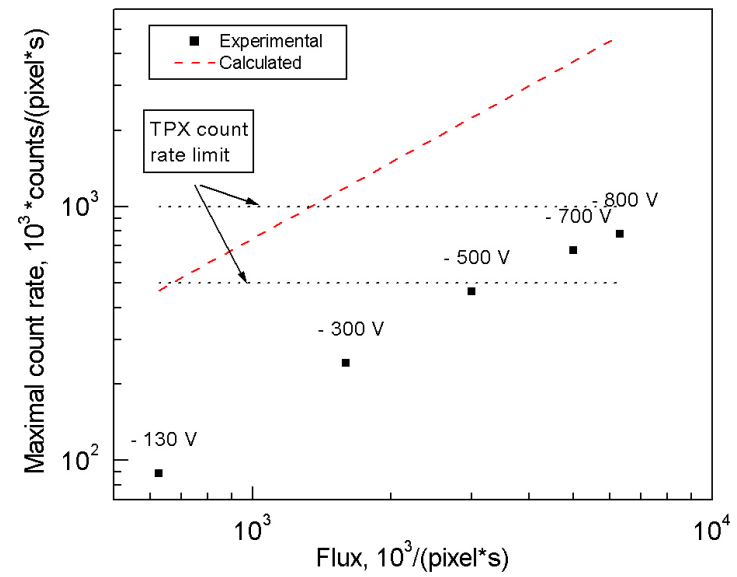

(b)

Figure 7. Dependence of the count rate of the $500 \mu \mathrm{m} \mathrm{GaAs}: \mathrm{Cr}+\mathrm{TPX}$ detector on the bias voltage (a) and $\mathrm{X}$-ray flux (b) for an X-ray tube voltage of $80 \mathrm{kV}$.

\subsubsection{Prototype GaAs:Cr imaging detectors}

Two types of prototype pixel GaAs:Cr sensors were produced and tested: $256 \times 256$ and $512 \times$ 768 pixels. The prototypes were compatible with Medipix 3/Timepix readout ASICs. The GaAs:Cr sensors were bump-bonded to Medipix/Timepix ASICs and tested at the FMF and IPS [4]. Images were obtained using a $512 \times 768$ pixel GaAs sensor (see figures 8 (a) and (b)). A simple flatfield correction was applied here (figure $8(\mathrm{~b})$ ) as well as a nearest neighbor interpolation for missing pixel lines between the Medipix chips and for the few non-counting pixels It is evident that the sensors provide good spatial resolution and enable high picture quality to be obtained. 


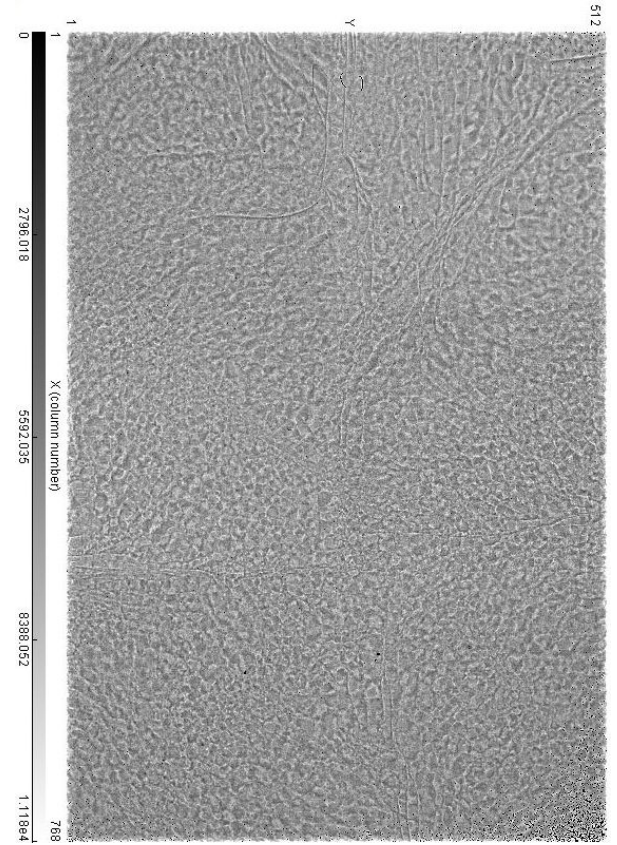

(a)

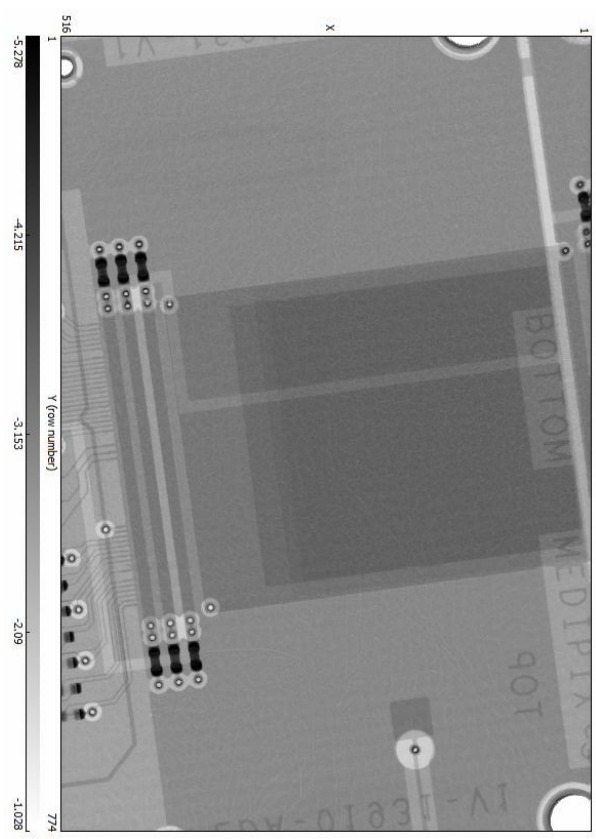

(b)

Figure 8. Images obtained with a $512 \times 76$ pixel GaAs sensor bump-bonded to Timepix ASICs: a flood image (a) and a test object image (b), a PCB board.

\section{Conclusions}

High charge collection efficiencies are shown to be obtainable by removing the surface layers on each side of a GaAs:Cr wafer.

It is found that for a bias voltage of $-200 \mathrm{~V}$ applied to a $500 \mu \mathrm{m}$ thick GaAs:Cr sensor, a linear dependence of the output signal on a exposure rate ranging between 0 and $400 \mathrm{mR} / \mathrm{s}$ and photon energies in the $40-100 \mathrm{keV}$ range is observed.

It is established experimentally that in the $40-80 \mathrm{keV}$ energy range, the maximum count rate of a GaAs:Cr Timepix detector amounts to $800 \mathrm{kHz} /$ pixel for a bias voltage of $-800 \mathrm{~V}$.

Prototype pixel X-ray imaging GaAs Timepix and Medipix detectors measuring $256 \times 256$ and $512 \times 768$ pixels were produced and tested. The sensors are shown to provide spatial resolution corresponding to the pixel pitch and to enable high-quality X-ray images to be obtained.

\section{Acknowledgments}

The experimental studies and preparation of the manuscript were financed in part by the Russian Foundation for Basic Research (Grant N 12-02-31187).

\section{References}

[1] G.I. Ayzenshtat et al., GaAs resistor structures for X-ray imaging detectors, Nucl. Instrum. Meth. A 487 (2002) 96. 
[2] A.V. Tyazhev et al., GaAs radiation imaging detectors with an active layer thickness up to $1 \mathrm{~mm}$, Nucl. Instrum. Meth. A 509 (2003) 34.

[3] L. Tlustos, G. Shelkov and O.P. Tolbanov, Characterisation of a GaAs(Cr) Medipix2 hybrid pixel detector, Nucl. Instrum. Meth. A 633 (2011) S103.

[4] E. Hamann et al., Characterization of photon counting pixel detectors based on semi-insulating GaAs sensor material, J. Phys. Conf. Ser. 425 (2013) 062015.

[5] A.N. Zarubin et al., Non-equilibrium charge carriers life times in semi-insulating GaAs compensated with chromium, IEEE SIBEDM (2006) 345.

[6] A.V. Tyazhev et al., GaAs radiation imaging detectors for nondestructive testing, medical, and biological applications, Proc. SPIE 5922 (2005) 59220Q.

[7] E. Hamann, Characterization of high resistivity GaAs as sensor material for photon counting semiconductor pixel detectors, Ph.D. Thesis, University of Freiburg (2013). 